\title{
Reactive oxygen species enhance differentiation of human embryonic stem cells into mesendodermal lineage
}

\author{
Ae-Ri $\mathrm{Ji}^{1,2 *}$, Seung-Yup $\mathrm{Ku}^{1,2 \star}$, Myung Soo $\mathrm{Cho}^{3}$, \\ Yoon Young $\mathrm{Kim}^{2}$, Yong Jin $\mathrm{Kim}^{1}$, Sun Kyung $\mathrm{Oh}^{2}$, \\ Seok Hyun Kim ${ }^{1,2}$, Shin Yong Moon ${ }^{1,2}$ and \\ Young Min Choi ${ }^{1,2,4}$ \\ ${ }^{1}$ Department of Obstetrics and Gynecology \\ Seoul National University College of Medicine \\ Seoul 110-799, Korea \\ ${ }^{2}$ Institute of Reproductive Medicine and Population \\ Medical Research Center \\ Seoul National University \\ Seoul 110-810, Korea \\ ${ }^{3}$ Research and Development Center \\ Jeil Pharmaceutical Co., Ltd., \\ Yongin 449-861, Korea \\ ${ }^{4}$ Corresponding author: Tel, 82-2-2072-2385; \\ Fax, 82-2-740-8956; E-mail, ymchoi@ snu.ac.kr \\ *These authors contributed equally to this work. \\ DOI 10.3858/emm.2010.42.3.018
}

Accepted 5 January 2010

Available Online 11 February 2010

Abbreviations: bFGF, basic fibroblast growth factor; BSO, buthionine sulfoximine; EB, embryoid body; hESCs, human embryonic stem cells; HNF, hepatocyte nuclear factor; SSEA, stage specific embryonic antigen

\begin{abstract}
Recently, reactive oxygen species (ROS) have been studied as a regulator of differentiation into specific cell types in embryonic stem cells (ESCs). However, ROS role in human ESCs (hESCs) is unknown because mouse ESCs have been used mainly for most studies. Herein we suggest that ROS generation may play a critical role in differentiation of $\mathrm{hESCs}$; ROS enhances differentiation of hESCs into bi-potent mesendodermal cell lineage via ROS-involved signaling pathways. In ROS-inducing conditions, expression of pluripotency markers (Oct4, Tra 1-60, Nanog, and Sox2) of hESCs was decreased, while expression of mesodermal and endodermal markers was increased. Moreover, these differentiation events of hESCs in ROS-inducing conditions were decreased by free radical scavenger treatment. hESC-derived embryoid bodies (EBs) also showed similar differentiation patterns by ROS induc-
\end{abstract}

tion. In ROS-related signaling pathway, some of the MAPKs family members in hESCs were also affected by ROS induction. P38 MAPK and AKT (protein kinases $B, P K B)$ were inactivated significantly by buthionine sulfoximine (BSO) treatment. JNK and ERK phosphorylation levels were increased at early time of BSO treatment but not at late time point. Moreover, MAPKs family-specific inhibitors could prevent the mesendodermal differentiation of hESCs by ROS induction. Our results demonstrate that stemness and differentiation of $h E S C s$ can be regulated by environmental factors such as ROS.

Keywords: cell differentiation; embryonic stem cells; mitogen-activated protein kinases; reactive oxygen species

\section{Introduction}

Human embryonic stem cells (hESCs) have a common characteristic; hESCs are capable of generating identical daughter cells by unlimited cell divisions and differentiating into all cell types (Carpenter et al., 2004). This ability of hESCs has been shown to be dependent on the intrinsic regulators and the extracellular environment niche. Recent reports have suggested that various extracellular ligands bind to their specific cell surface receptors, and the ligand-receptor interactions activate diverse intracellular signal transductions (Rhee, 1999). Activated intracellular signaling pathways are amplified by secondary messengers, thus managing the expression of transcription factors that induce cell responses, such as proliferation, differentiation, and apoptosis (Sohal et al., 1986). Recently, reactive oxygen species (ROS), which are free radicals, have been shown to be secondary messengers in cell signal transduction (Rhee, 1999). ROS generated at low-to-moderate concentrations act as signaling molecules that are involved in signal transduction-mediated biological effects in cells. Such effects of ROS are related to differences in the concentrations of ROS (Valko et al., 2007).

ROS are generated by signal transduction cascades of various growth factor-, cytokine-, and hormone-mediated pathways and then induce individual cellular responses in non-phagocytic cells (Thannickal and Fanburg, 2000). Moreover, 
A
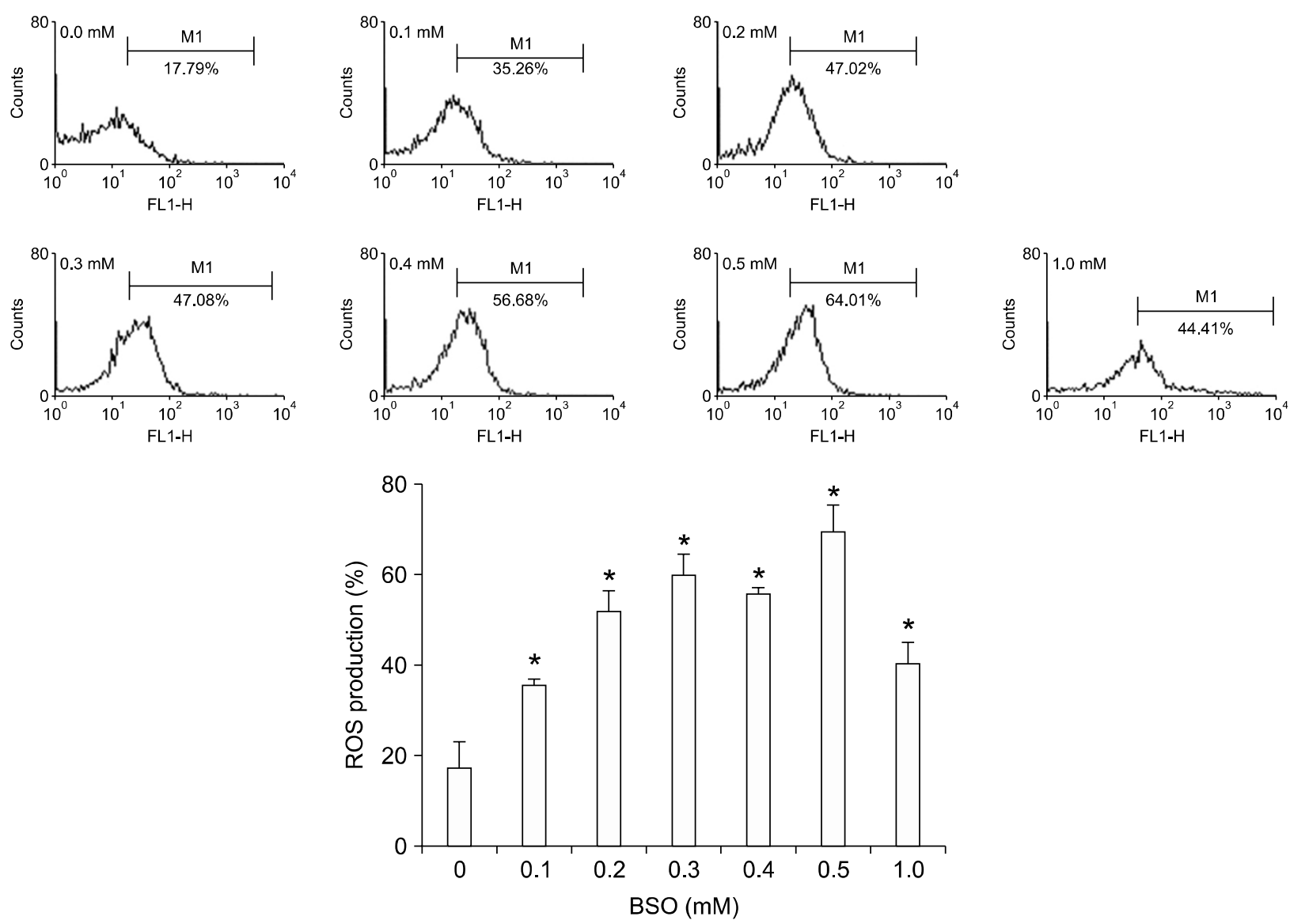

Figure 1. Generation of endogenous ROS in hESCs by buthionine sulfoximine (BSO), generated ROS was measured by staining with 2', 7'dichlorofluorescene acetate (DCF-DA). (A) Endogenous ROS measurement. Treatment in vitro with BSO increased levels of intracellular ROS in a dose-dependent manner. Fluorescent DCF was monitored by FACS analysis. ${ }^{*} P<0.05$, significantly different from the untreated controls $(n=3)$. (B) Apoptosis analysis on response to elevated ROS levels in hESCs. Cells were cultured in DMEM/F12-containing BSO in a dose-dependent manner. BSO at a high concentration induced apoptosis of hESCs, which increased the number of dead and floating cells. Representative flow cytometry data are shown annexin V incorporation (FL1) and PI staining (FL2) $(n=3) .{ }^{*} P<0.05$, significantly different from the untreated controls.

various growth factors that bind to receptor tyrosine kinases (RTKs) have been reported to induce endogenous ROS. Shibanuma et al. (1990) reported that PDGF stimulates cellular production of $\mathrm{H}_{2} \mathrm{O}_{2}$, which is thought to be a competence factor in BALB/3T3 cell proliferation. PDGF also induces the signal transducer and activator of transcription (STAT) family via a $\mathrm{H}_{2} \mathrm{O}_{2}$-dependent mechanism (Simon et al., 1998).

Interestingly, recent studies on the effects of ROS in mouse ESCs (mESCs) have increased; ROS have been reported that it play a crucial role in cell differentiation to regulate mESCs (Sauer and Wartenberg, 2005; Valko et al., 2007). Previous studies have reported the function of ROS in mESCs differentiation into cardiomyocytes; ROS promotes differentiation toward both cardiomyo- genic and vascular cell lineages in mESCs (Sauer and Wartenberg, 2005). In mESCs, increasing evidence implies that cardiomyogenesis is directly influenced by ROS through the MAPKs family (Sauer and Wartenberg, 2005). The ROS-activated MAPKs family consists of ERK $1 / 2$, JNK and p38 MAPK, which play a crucial role in cell differentiation and have been reported to regulate ESCs (Sauer and Wartenberg, 2005; Valko et al., 2007). Hypoxia regulate the expression of cardiotrophin-1 (CT-1), and hypoxia induced factor-1 (HIF-1) were increased during cardiomyogenesis of differentiating mESCs by the ROS-related MAPKs family (Ateghang et al., 2006). ROS-induced differentiation was stimulated by a ROS-modulated signaling pathway in mESCs (Sauer et al., 2000; Sauer and Wartenberg, 2005; Ateghang et al., 2006). 
B
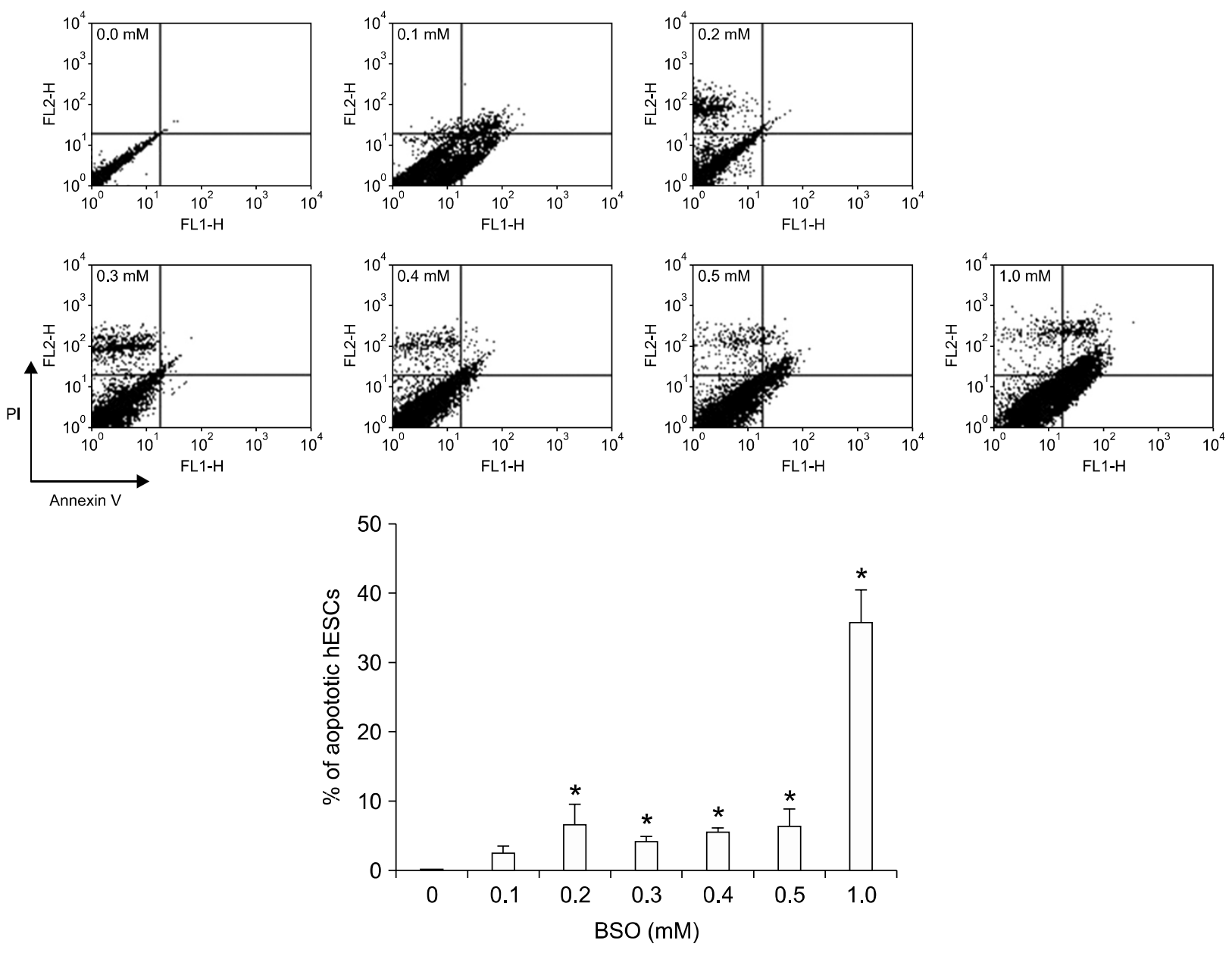

Figure 1. Continued.

To date, previous studies have identified the effects of ROS in mESC differentiation; ROS stimulate the differentiation of mESCs into specific lineages by a ROS-modulated signaling pathway (Sauer and Wartenberg, 2005; Ateghang et al., 2006). However, the importance of ROS in the in vitro differentiation of $\mathrm{hESCs}$ into specific lineages has not been underestimated. In the current study, we investigated the changes in hESC pluripotency in a ROS-induced system. We demonstrated the diminished pluripotency of hESCs induced by continuous exposure to ROS. Moreover, we suggest a possible role of ROS in hESC differentiation; ROS enhances differentiation of hESCs into bi-potent mesendoderm that can specify both mesoderm and endoderm (Tada et al., 2005) via the activation of MAPKs family and/or AKT signal transduction by ROS.

\section{Results}

\section{Generation of endogenous ROS in hESCs}

BSO which inhibits intracellular glutathione and enriches the level of ROS was used for ROS induction in hESCs and generated ROS were detected by DCF-DA staining. DCF-DA is trapped within cells by esterification and converted in its fluorescent form by reaction with peroxides (Bunting, 2002). Endogenous ROS was generated dose-dependently in hESCs and was peaked at $0.5 \mathrm{mM}$ (Figure 1A) without serious apoptosis (Figure 1B). Serious apoptosis was observed at a high concentration (1 mM) of BSO (Figure 1B) and the ROS level was also decreased slightly (Figure 1A). We performed apoptosis analysis to determine the moderate ROS-induced conditions. At the high concentration, hESCs had an increased number of dead and floating cells from day 2 after 

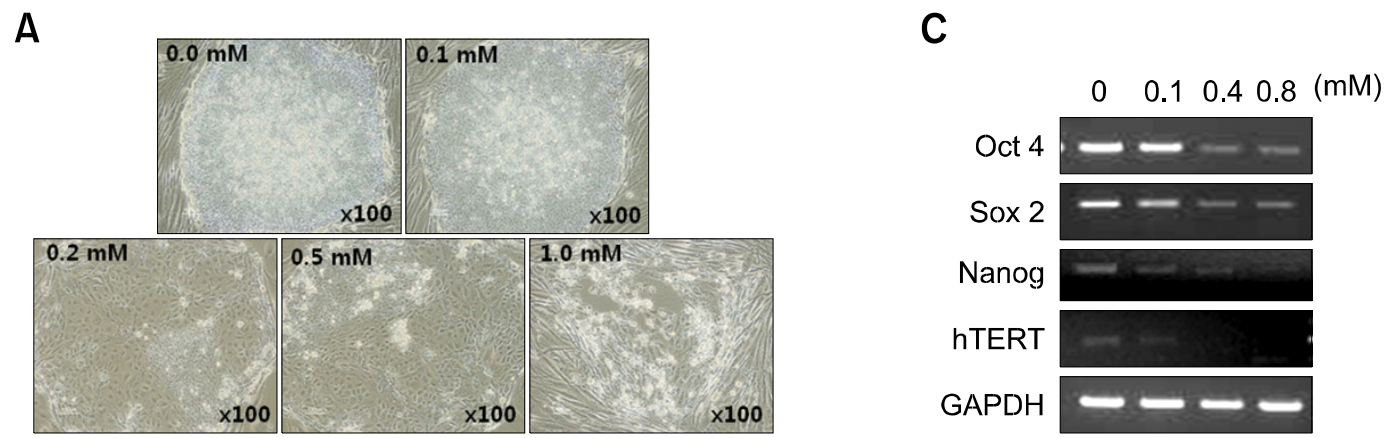

B
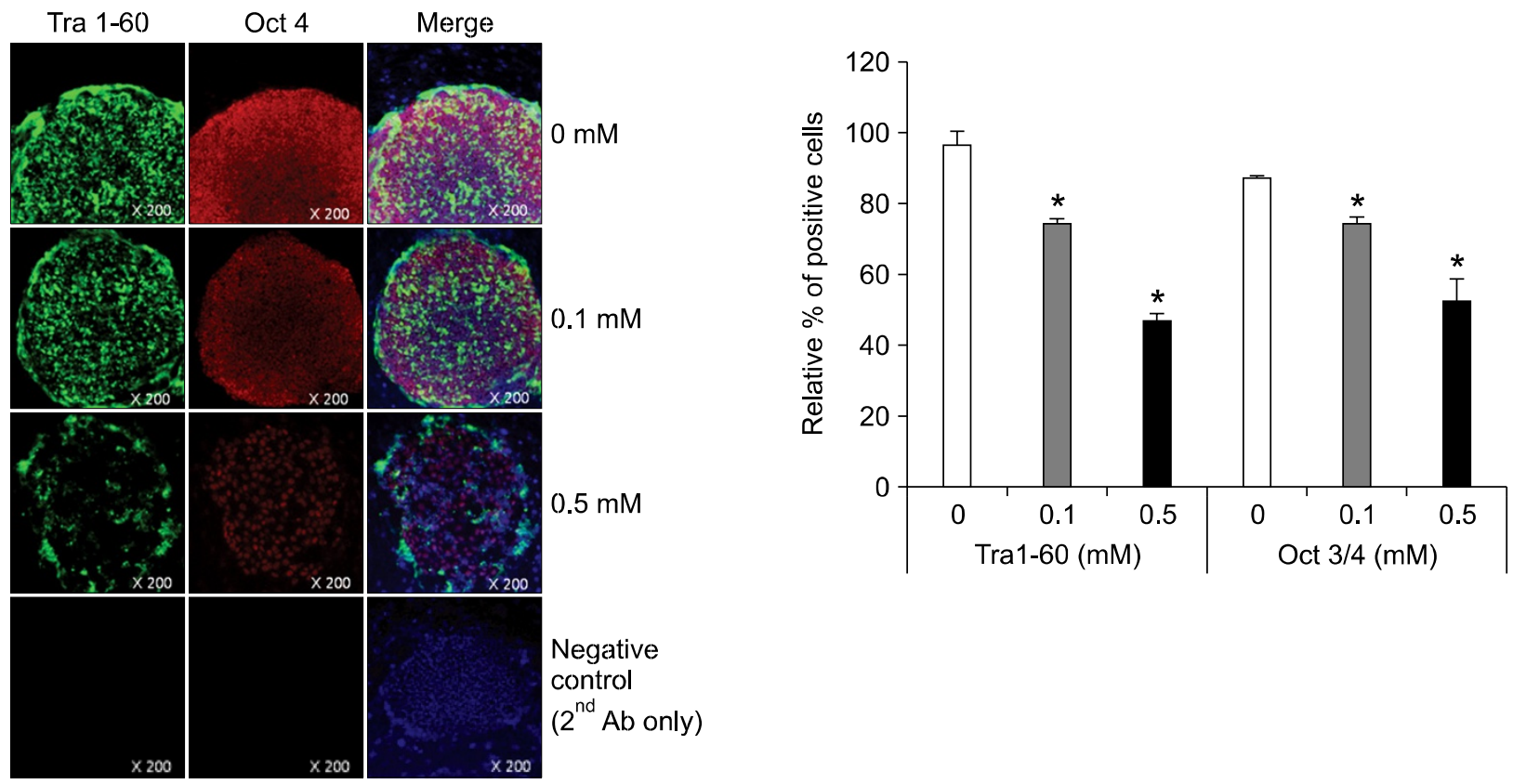

Figure 2. Loss of pluripotency in ROS-induced hESCs. Increased ROS abrogates the maintenance of stemness in hESCs. Cells were grown in media with or without BSO. Then, we observed the morphology of hESCs through day 5. (A) Phase contrast microscope images represented a compact hESC colony at $0.0 \mathrm{mM}$ as a control. Human ESCs exposed to BSO were shown differentiated form. Morphologies of ROS-induced hESCs lost the undifferentiated shape. Microscopic data represented differentiation of hESCs increasing the concentration of BSO. (B) Diminished expression of hECS markers by ROS is shown by a confocal microscope. Cells were grown in $35 \mathrm{~mm}$ dishes for 5 days, followed by immunocytochemisty using anti-Tra $1-60$ and anti-Oct 4 antibodies. Downregulation of hESC markers (Oct 4 and Tra-1-60) shown in a dose-dependent manner. The graph was representative average of positive cells. ${ }^{*} P<0.05$, significantly different from the untreated control. (C) Decreased expression of pluripotency markers in ROS-elevated hESCs. Representative RT-PCR data are shown down-regulation of pluripotent markers using cDNA of undifferentiated hESCs and ROS-induced hESCs samples for 5 days cultured in a dose-dependent manner of BSO. GAPDH was internal controls.

treatment with BSO. The percentage of apoptotic cells at $1 \mathrm{mM}$ had increased 3 or 4 -fold $(n=3$ for each experimental condition; Figure 1B) compared with the low concentration groups $(0.1-0.5 \mathrm{mM})$ and up to 20 -fold on different experiments (data not shown).

\section{Down-regulated expression of pluripotency markers in hESCs by ROS}

To examine the biological effects of ROS on the pluripotency of hESCs, the cells were treated with BSO in a dose-dependent manner. Human ESCs are characterized by a high ratio of nucleus to cytoplasm. Treatment of ROS induced differentiation of hESCs, as characterized by morphologic changes and decreased expression of pluripotency markers. Phage contrast images showed that at 0 $\mathrm{mM}$ BSO as a control, hESC colonies were densely packed cells with smooth outlines and no differentiated cells; however, BSO-induced hESC differentiation and no compact morphology in a 
A
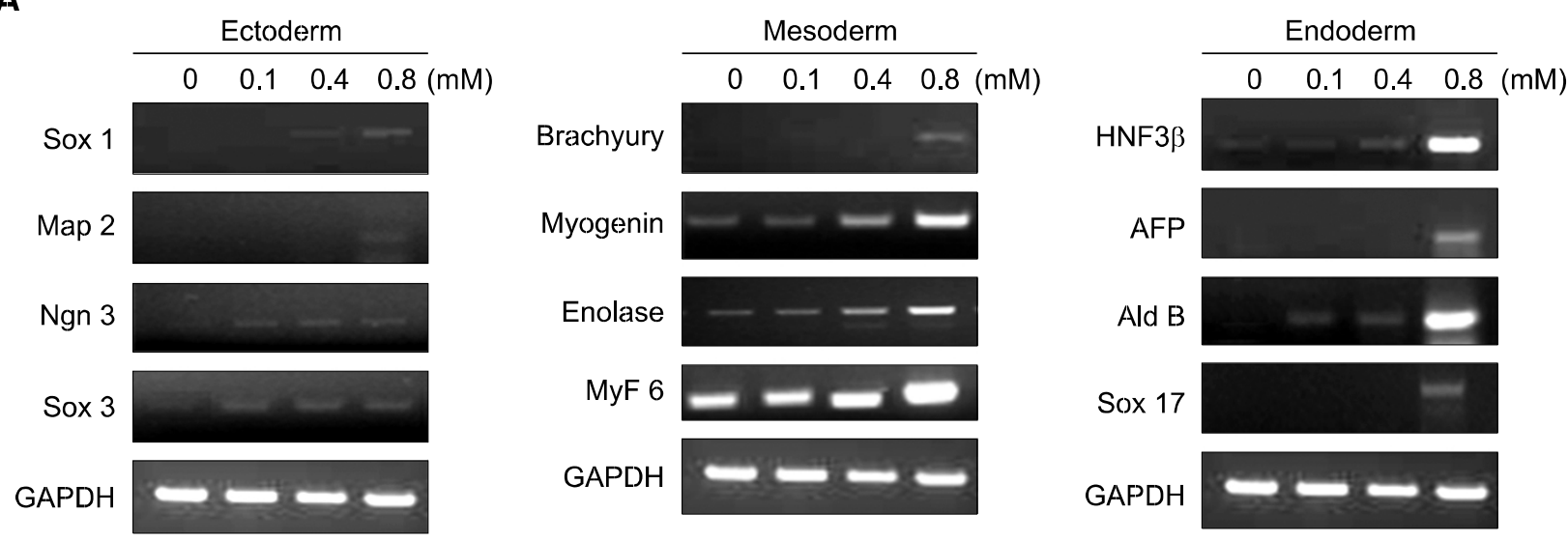

B
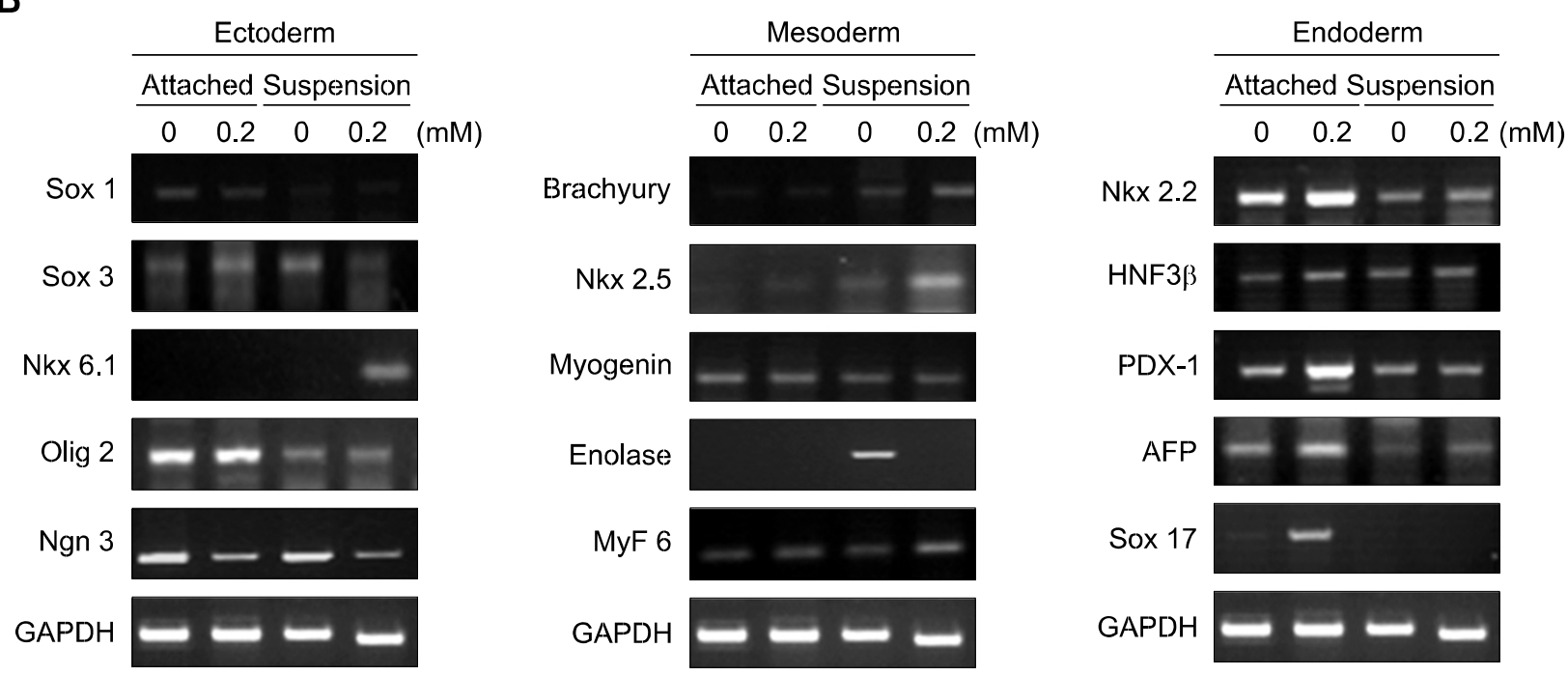

Figure 3. Effect of ROS on hESC or hESC-derived EB differentiation. ROS induces differentiation of hESCs in ROS-elevated condition. Cells were treated with BSO in a dose-dependent manner. Treatment of BSO induced differentiation as characterized by RT-PCR at the molecular level. (A) Up-regulation of three germ layer genes in hESCs. The genes expression were increased in a dose-dependent of BSO. Particularly, gene expressions of mesoderm and endoderm were increased in hESCs. (B) Expression of differentiated markers in EBs. Human ESC-derived EBs was grown in EB medium for 5 days. Then EBs was maintained in the absence (control) or presence of $0.2 \mathrm{mM} \mathrm{BSO}$ for additional 5 days. The cells expressed represented genes. EBs on attached cultures were mainly increased Nkx2.2, HNF3 $\beta$, PDX-1, and Sox17 and EBs in suspension cultures promoted gene expression for both mesoderm (brachyury and Nkx2.5) and endoderm (Nkx2.2 and HNF3 $\beta$ ) as compared with controls.

concentration-dependent manner (Figure 2A). During differentiation, hESCs flattened and lost indigenous hESC morphology. Loss of pluripotency was confirmed at the molecular level by immunocytochemisty and RT-PCR analysis. As shown in Figure 2B, the hESC markers, Oct 4 and Tra 1-60, were strikingly decreased in hESCs growing in ROS-induced conditions compared with the control group. We observed the down-regulation of the pluripotency markers, Sox2, Nanog, hTERT, and Oct 4, by RT-PCR (Figure 2C). Our data suggest that the transcripts of these pluripotent markers were also decreased in hESCs by ROS.

\section{Up-regulated differentiation in hESCs into the both mesodermal and endodermal lineage by ROS}

Next, to analyze the function of ROS on differentiation, we investigated the expression of the three germ layer markers. The cells were treated with BSO in a dose-dependent manner. ROS induced the differentiation of hESCs, as determined by expression of embryonic lineage markers. The differentiated shape of hESCs was shown within 1 day (data not shown). To establish the expression of selected differentiation markers, RT-PCR was performed (Figure 3). Differentiation to various em- 
A
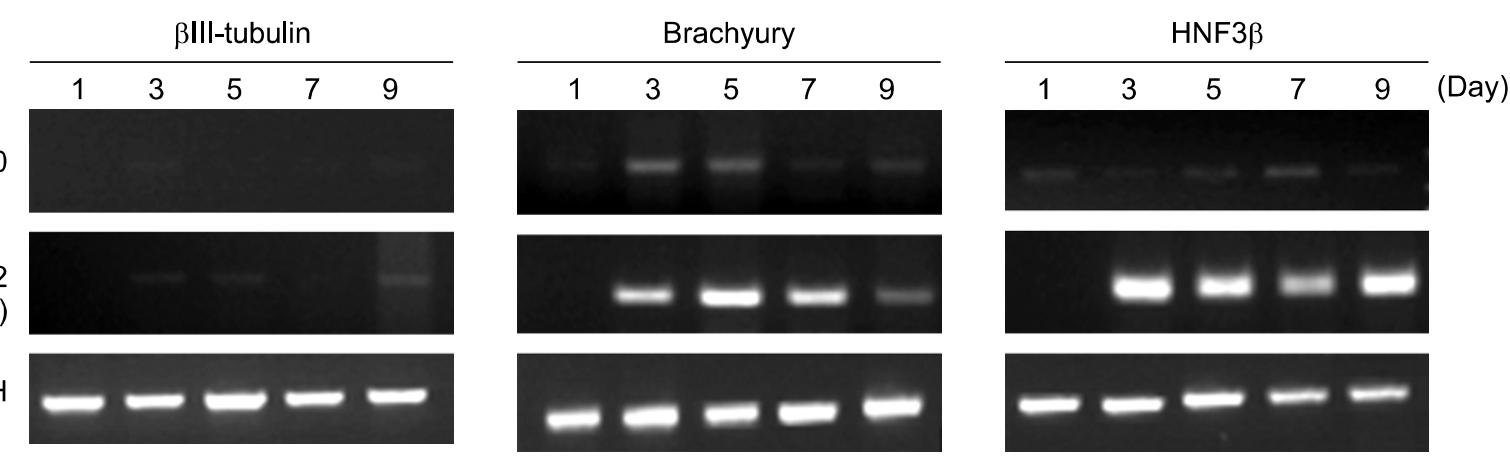

B

0.2

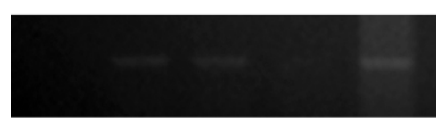

GAPDH
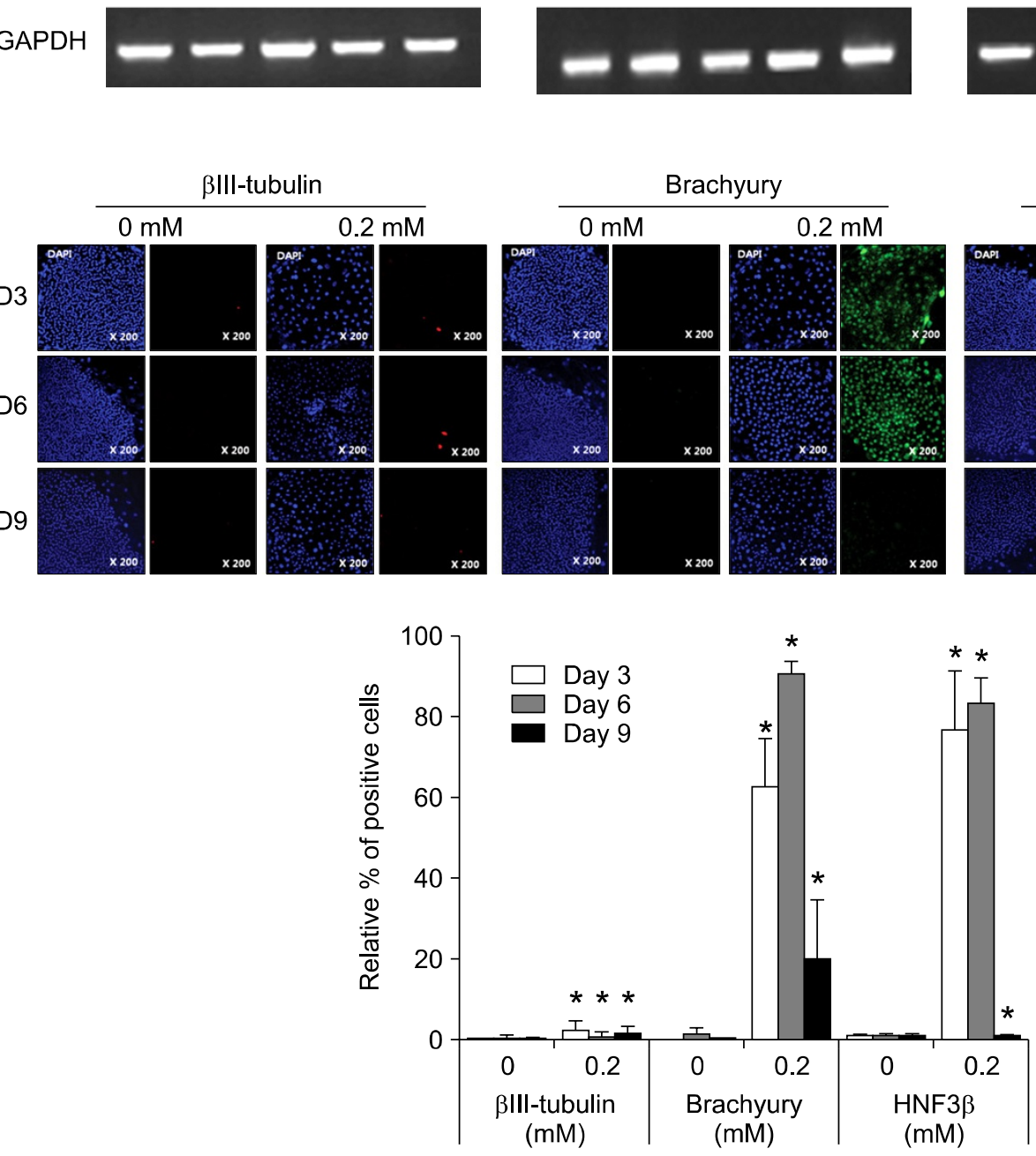

Figure 4. Course of differentiated marker expression after ROS treatment in hESCs. Comparison of marker expressions with hESCs at 1, 3, 5, 7, and 9 days in the ROS-induced conditions. (A) RT-PCR analysis of gene expression in hESCs grown in medium containing with or without BSO. Total RNA was extracted from each hESC sample and subjected to RT-PCR analysis with a set of specific primers. Gene expression of brachyury and HNF3 $\beta$ were up-regulated in hESCs grown in ROS-elevated conditions. (B) Expression of lineage specific markers in ROS-induced hESCs is day-dependent. Double staining was governed on hESCs cultured during days 3,6 , and 9 in ROS-induced conditions with antibodies against $\beta$ III-tubulin, brachyury, and HNF3 $\beta$. The graph was representative average of positive cells. ${ }^{*} P<0.05$, significantly different from the untreated control.

bryonic lineages was confirmed by up-regulation of Sox1, Ngn3, and Sox3 (ectoderm), brachyury, myogenin, and myogenic factor 6 (MyF6; mesoderm), and HNF3 $\beta$, AFP, and Sox17 (endoderm). The expressions of mesoderm- or endoderm-related genes were significantly up-regulated in a dose-dependent manner of BSO, rather than ectoderm-related genes (Figure 3A). These data indicated that ROS promoted hESC differentiation into bi-potent mesendoderm cells, even in the presence of FGF 2, which is the main factor to sustain the undifferentiated state of hESCs. So, we 


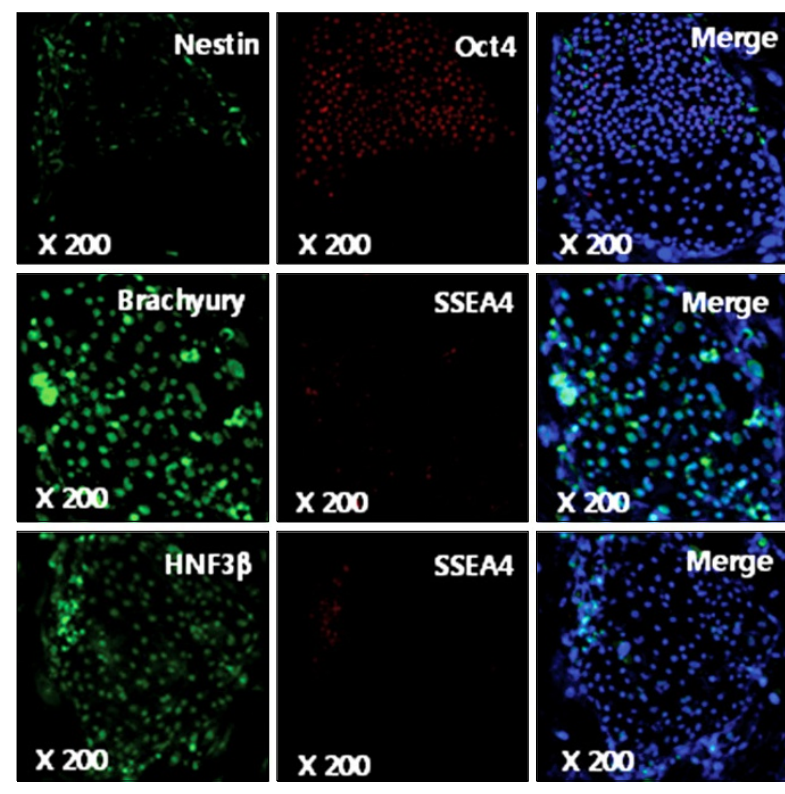

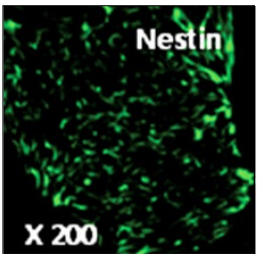
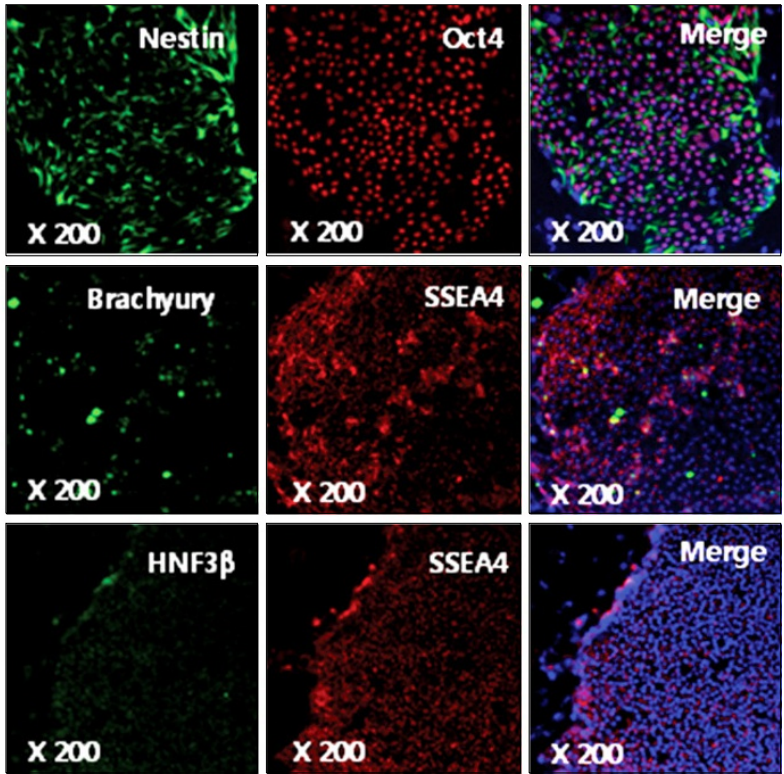

BSO

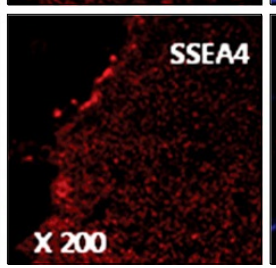

BSO + Vit. C

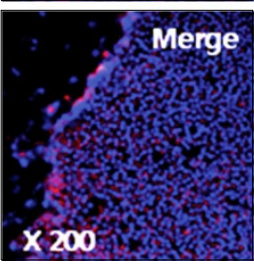

$\times 20$

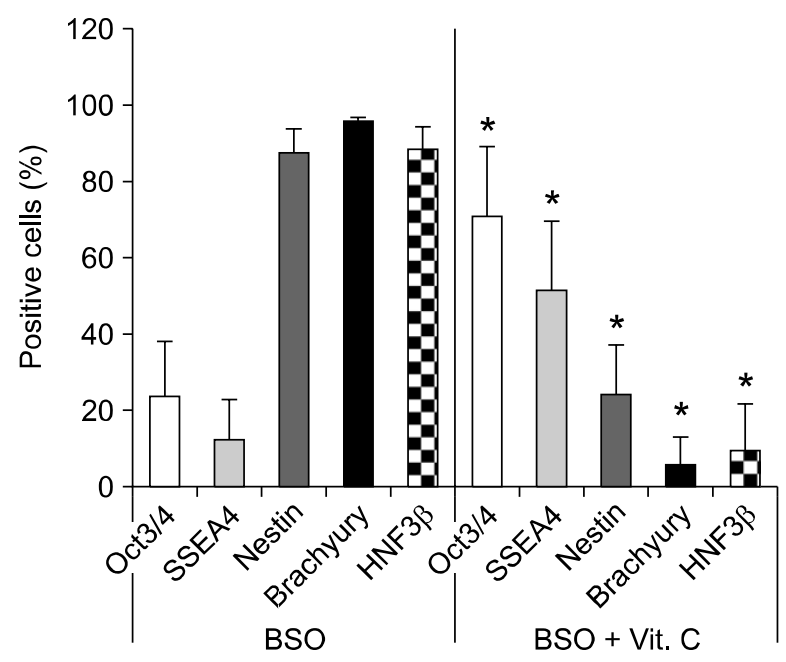

Figure 5. Effects of free radical scavengers on ROS-induced differentiation. Two-day-old hESCs after transfer were incubated with or without $100 \mu \mathrm{M}$ vitamin $C$ for 4-5 days. Free radical scavenger was presented in the cell culture medium until inspection. Note, that in the presence of free radical scavengers, ROS-stimulated differentiation was significantly inhibited, but restored hESC markers (Oct4 and SSEA4). This data point suggested involvement of ROS. The cells were counted as positive cells for each marker. The graph was shown as the percentage change in $\mathrm{hESCs}$. ${ }^{*} P<0.05$, significantly different from the scavenger-untreated controls.

further investigated the ROS effects in EBs, which are differentiated cells corresponding to a precursor.

\section{Changes of gene expression in EBs by ROS in vitro culture}

To test the influence of ROS in EB outgrowth, EBs were cultured in EB media (hESC culture mediumreduced FGF 2). Human ESCs were detached from culture dishes by $2.5 \mathrm{mg} / \mathrm{ml}$ of collagenase IV, and the aggregates of hESCs were cultured in EB media for 5 days. The diagnosis of differentiation in EBs differs in accordance with the culture method. We then grew the spontaneously differentiated EBs by suspension or attached cultivation with $(0.2$ $\mathrm{mM}$ ) or without BSO for additional 5 days. As shown in Figure 3B, EBs on attached cultures were mainly increased Nkx2.2, HNF3 $\beta, P D X-1$, and Sox17, which is an endoderm lineage in ROS-elevated conditions. Moreover, EBs in suspension cultures promoted gene expression for both mesoderm (brachyury and Nkx2.5) and endoderm 
A
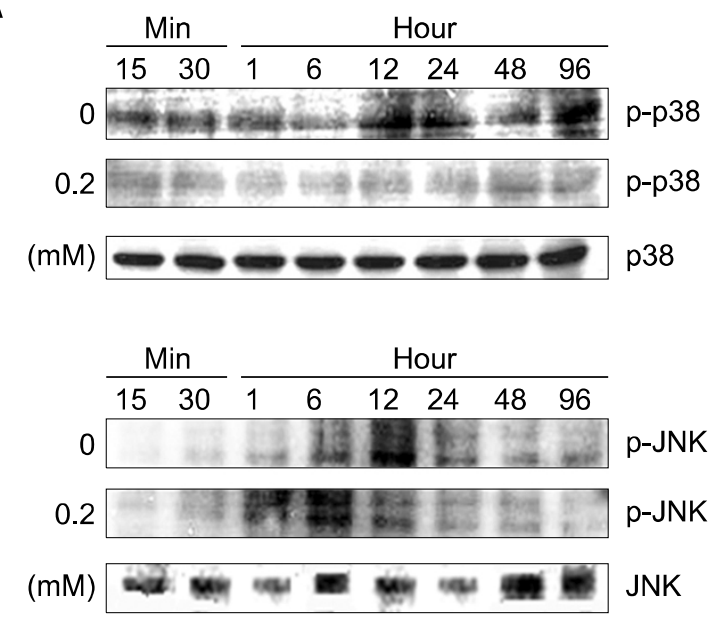

B

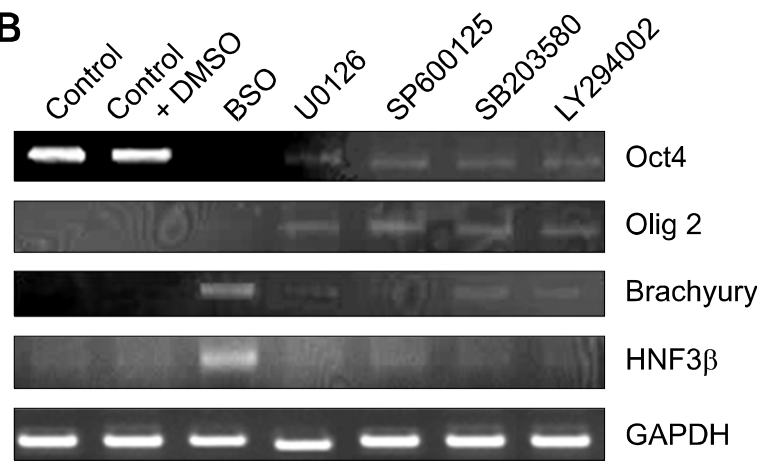

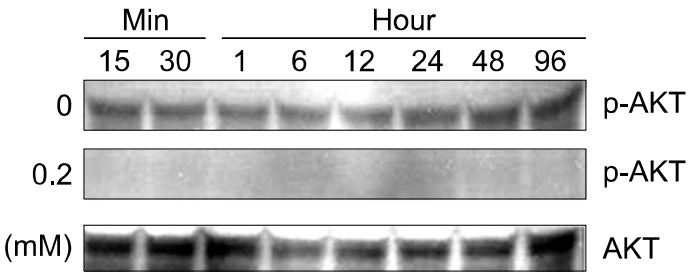

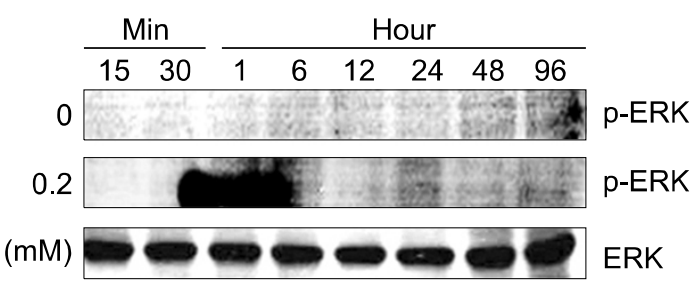

Figure 6. The activation of four distinct protein kinase cascades and the change of gene expression by specific inhibitor. (A) Western blots demonstrating synchronous phosphor-activation of MAPKs family initiated by endogenous ROS in hESCs. Antibodies recognize phosphorylated residues. Cells were exposed to ROS in time-dependent manners, lysed, electrophoresed on $9 \%$ or $12 \%$ polyacrylamide gels, and probed with the appropriate phosphorylation-state specific primary antibody. (B) Effects of MAPKs family inhibitors on the expression of hESC marker (Oct4) or lineage-specific markers (olig2, braychyury, and HNF3 $\beta$ ). The data are presented as relative change of gene expression as compared to the untreated control.

(Nkx2.2 and HNF3 $\beta$ ) as compared with controls. The present results demonstrated that ROS induced differentiation in both undifferentiated hESCs and EBs into mesoderrmal and endodermal lineages.

We also observed the expression of genes in accordance with days. The specific genes were detected as early as day 1 for mesodermal or endodermal lineages in hESCs and reached a significant level from days 3-7 (Figure 4A). Moreover, immunocytochemistry analysis showed that on day 3 , the specific marker-positive cells appeared for brachyury or HNF3 $\beta$, but not $\beta$ III-tubulin, and on day 6 , the maximal expression was shown $(n=3$ for each experimental condition; Figure 4B). Therefore, we suggest that ROS can commit differentiation of hESCs or EBs to a specific mesendoderm lineage.

\section{Effect of free radical scavenger on induced differentiation of hESCs by ROS}

We perceived that ROS induced differentiation of hESCs into the mesendodermal lineage. Whether inhibition of ROS generation can obstruct the biological changes in hESCs was assessed, and the cells in ROS-induced conditions were subjected to a free radical scavenger. To investigate the association of ROS and stimulated differentiation, hESCs were incubated with the free radical scavenger, $100 \mu \mathrm{M}$ vitamin $\mathrm{C}$, with $0.2 \mathrm{mM}$ BSO. The maximum effect of scavenger was shown at $100 \mu \mathrm{M}$; however, at $50 \mu \mathrm{M}$ there was no effect for blocking the differentiation of hESCs (data not shown), and hESCs had increased cell death at a higher concentration $(200 \mu \mathrm{M}$; data not shown). The free radical scavenger was continuously exposed to hESCs in ROS-induced conditions for $4-5$ days. The free radical scavenger 
influenced the translation of differentiating or undifferentiating markers. Treatment of scavenger decreased the expression of brachyury and HNF3 $\beta$ and increased the expression of the pluripotency marker, Oct 4, as well as stage-specific embryonic antigen-4 (SSEA 4) in hESCs. As shown in Figure 5, the expression of brachyury and HNF3 $\beta$ were diminished in hESCs by free radical scavenger. Comparably, hESC markers (Oct4 and SSEA4) were increased in the presence of free radical scavenger ( $n=3$ for each experimental condition; Figure 5). We know that the free radical scavenger inhibited the induced-differentiation of hESCs by ROS.

\section{Activation of MAPKs family by ROS in hESCs}

Previous studies have reported that high activity of the MEK/ERK signaling pathway promotes differentiation of mESCs and hESCs (Burdon et al., 2002). In the other study, PI3K/AKT and MAPK/ERK pathways are decreased upon differentiation of hESCs (Armstrong et al., 2006). In this study, we observed that ROS promoted the differentiation of hESCs into the mesendodemal lineage. To understand the molecular mechanism of the hESC differentiation induced by ROS, we focused on the MAPKs family as a main target signaling pathway of ROS. Whether the MAPKs family is involved in hESC differentiation, activation of these signaling pathways was examined in a time course. Even though at low concentration of BSO $(0.2 \mathrm{mM})$, p38MAPK and AKT phosphorylation levels were significantly decreased in hESCs culture. JNK and ERK phosphorylation levels were increased at early time of BSO treatment but not at late time point (Figure 6A). These results suggest that activation of MAPKs is influenced by BSO and ROS can be key regulator on the activation of MAPKs family.

To investigate the role of MAPKs family in hESCs commitment, we analyzed the effects of specific inhibitors in ROS-induced hESCs. $10 \mu \mathrm{M}$ MEK (upstream of ERK) inhibitors, U0126; $1 \mu \mathrm{M}$ JNK inhibitor, SP600125; $1 \mu \mathrm{M}$ p38MAPK inhibitor, SB203580 and $50 \mu \mathrm{M}$ PI3K/AKT inhibitor, LY294002 were treated in ROS-induced hESCs for 2 days, respectively. ROS-induced brachyury and HNF3 $\beta$ expressions were decreased in hESCs by all used inhibitors. On the other hand, ectodermal gene, Olig2 expression was increased (Figure 6B). These results suggest that ROS can regulate the hESC differentiation via MAPKs signaling.

\section{Discussion}

In this study, we have described the biological effects of ROS on hESC differentiation, particularly into mesendodermal lineages. This study is the first trial to determine the responses of hESCs in ROS-induced conditions. Furthermore, we demonstrated the down-regulation of pluripotency and up-regulation of differentiation into a mesendodermal lineage in hESCs as well as hESC-derived EBs.

Previous studies have reported that differentiation into specific cell types from mESCs may be facilitated by ROS (Ateghang et al., 2006; Buggisch et al., 2007; Ding et al., 2008; Lange et al., 2009). ROS was generated by a growth factor related-specific process (Lange et al., 2009) or NADPH oxidase (Sauer et al., 1999) in mESC-derived EBs that induced differentiation, especially into cardiomyocytes or endothelial cells. These studies have shown the significance of ROS function in inducing differentiation of mESCs. In this study, we first characterized the ROS effect on pluripotency of hESCs. For this purpose, we used BSO to induce ROS-elevated conditions (Ito et al., 2006). As a result, loss of pluripotency was observed in hESCs by an elevated level of ROS; we confirmed the significant down-regulation of Oct4, Sox2, Nanog, and Tra-1-60, as well as hTERT. We propose that ROS induced decreased pluripotency of hESCs, indicating induction of differentiation of hESCs. The cells were morphologically differentiated by ROS, even though hESCs were grown in ES media containing $4 \mathrm{ng} / \mathrm{ml}$ of FGF 2. Considering that exogenous FGF 2 has been used as an important factor in maintaining the undifferentiated state of hESCs (Dvorak et al., 2005), these results indicated that ROS affected hESC pluripotency, overcoming the effects of FGF 2.

A role for ROS in embryogenesis has been reported for the past decade. Various studies have suggested that ROS play a determining role in cellular differentiation (Sohal et al., 1986). In our current study it was shown that differentiation of hESCs can be accelerated by ROS within 1 day; the cells changed their morphology into a differentiated form, the expression of both early mesoderm- and endoderm-specific markers were up-regulated and pluripotency markers were down-regulated. Therefore, we suggest that ROS is involved in inducing the differentiation of hESCs. Maximal effects were achieved at the concentration of BSO of as low as $0.5 \mathrm{mM}$ without apoptosis which was observed at days 3-6. We also assessed the effects of ROS in hESC-derived EBs. The differentiation of EBs differs according to the culture method. When cultured by suspension or attached cultivation, EBs tended to express the 
mesoderm or endoderm, respectively. We considered that ROS act as a signaling molecule and that induce the differentiation of hESCs as well as EBs into specific cell lineages. Our results correspond to previous studies in mESCs (Sauer and Wartenberg, 2005; Ateghang et al., 2006; Buggisch et al., 2007). Additionally, we observed that the free radical scavenger inhibited the induced-differentiation of hESCs by ROS, which strongly suggests the role of ROS in the triggering of hESCs differentiation.

In this study, we observed the activation of the MAPKs family and AKT signal transduction in hESCs by ROS. It has been previously reported that ROS production mediates on signaling pathway (Thannickal and Fanburg, 2000). In ligand-receptor-mediated, intracellular signaling is commonly promoted by ROS. ROS acts primarily by maintaining phosphorylation of signal molecules in intracellular signaling pathways (Allen and Tresini, 2000). Exogenously- or endogenously-produced ROS activate the MAP kinases family such as ERK, JNKs and p38MAPK. In our study, hESCs as a control group were cultured in media without FGF 2 for 2-4 weeks, in order to prevent the possible phosphorylation of MAPK family and AKT by FGF 2. The phosphorylation of ERK1/2 and JNK were elevated in early time point and p38MAPK and AKT were down-regulated by ROS in hESCs, regardless of the presence of FGF 2. We suggest that ROS can regulate the hESC differentiation via MAPKs signaling. We used BSO to induce ROS generation in hESCs. BSO increased the ROS levels at low concentrations (0.1-0.5 mM), whereas increased apoptosis occurred at a high concentration (1.0 mM). Presumably, a higher concentration of BSO increases excessive endogenous ROS causing oxidative stress, which is detrimental to hESCs. Therefore, the concentrations of between 0.1-0.5 mM were used for ROS production. These concentrations of ROS have been also employed in another previous study that used hematopoietic stem cells (Ito et al., 2006). Our results may provide a possible new comprehension of ROS effects and molecular mechanism of ROS-induced signal transductions in hESCs. We plan to study the differentiation of hESCs into specific cell types by ROS. Also, the key downstream effectors of the MAPKs family and AKT signaling pathways remain to be further studied in hESCs.

The limitations of this study are as follows. We did not observe the effect of ROS for long-term culture in hESCs or EBs. Because hESCs were cultured on mitotically-inactivated STO, which are best maintained for a week as feeder cells, so
hESCs increased spontaneous differentiation when grown for more than 1 week. Therefore, we investigated the effects of ROS in hESCs around 7 days to eliminate spontaneous differentiation of hESCs. In EBs, we showed increasing cell death in spite of low concentration of BSO when EBs were grown for more 2 weeks in ROS-induced conditions. Because of these technical limitations, we performed the experiments for ROS effects in hESCs for a short-term culture.

\section{Methods}

\section{Human ESC cultures}

The human embryonic stem cell lines, SNUhES3 (passage 27-80) and SNUhES11 (passage 30-80), were used, and the undifferentiated hESCs were maintained as described in previous report (Oh et al., 2005). In each experiment, hESCs were transferred to $35-\mathrm{mm}$ tissue culture dishes; 2 days thereafter, the cells were grown in the presence of buthionin sulfoximine (BSO; Sigma-Aldrich) in a dose-dependent manner. Under these conditions, hESCs were removed from the culture dishes mechanically on day 7 (BSO treatment for 5 days) for measurement of the ROS levels.

\section{ROS induction and inhibition}

Human ESCs were treated with BSO in a dose-dependent manner (0.1-1.0 mM) from 2 days after transfer. The media containing BSO were changed every $24 \mathrm{~h}$ for 5 days. On day 5 , hESCs were detached by a mechanical method from STO feeder cells, and trypsinized by diluted 0.025 $\mathrm{mM}$ trypsin-EDTA to single cells. After washing, the cells were incubated with $10 \mu \mathrm{M}$ 2',7'-dichloroflurescein diacetate (DCF-DA; Sigma-Aldrich) for $30 \mathrm{~min}$ in a $37^{\circ} \mathrm{C}$ water bath in darkness. The production of ROS was measured by a FACSCalibur flow cytometer (Becton, Dickinson and Company, Franklin Lakes, NJ, http://www.bd.com) using a CellQuest program. In parallel experiments, ROS were scavenged by $100 \mu \mathrm{M}$ vitamin C (Sigma-Aldrich).

For MAPK or AKT inhibition, U0126 (10 $\mu \mathrm{M}$, MEK inhibitor), SP600125 (1 $\mu \mathrm{M}$, JNK inhibitor), SB203580 (1 $\mu \mathrm{M}$, p38MAPK inhibitor), or LY294002 (50 $\mu \mathrm{M}$, PI3K/AKT inhibitor) were treated for 2 days with BSO.

\section{Measurement of intracellular ROS}

DCF-DA is a non-specific probe for ROS to produce fluorescent 2'-7'-dichlorofluorescin. The acetate groups of the DCF-DA are cleaved in vivo by indigenous esterases, and subsequent oxidation by intracellular peroxides produces the fluorescent DCF-DA (Thannickal and Fanburg, 2000), which can be detected by flow cytometry. Human ESCs were mechanically detached from culture dishes. Colonies of hESCs were treated with trypsin/EDTA to make single cells. Cells were washed with Krebs-Ringer-HEPES (KRH) buffer containing $1 \mathrm{mM}$ calcium, $0.1 \mathrm{mM}$ glucose, and $0.2 \%$ BSA. These cell 
suspensions were incubated with $10 \mu \mathrm{M}$ DCF-DA in a $37^{\circ} \mathrm{C}$ water bath for $30 \mathrm{~min}$. To prevent degradation of DCF-DA from lights, we performed the reaction in the darkness.

\section{Immunocytochemistry}

Immunocytochemistry was performed as described in previous report (Park et al., 2008). The cells were incubated with the following primary antibodies: rabbit anti-Oct 4 antibody (1:400; Santa Cruz Biotechnology, Inc., Santa Cruz, CA, http://www.scbt.com), mouse anti-Tra-1-60 (1:400; Chemicon, Temecula, CA, http://www.millipore.com), rabbit anti-brachyury (1:200; Santa Cruz Biotechnology, Inc.), rabbit anti-HNF3 $\beta$ (1:200; Santa Cruz Biotechnology, Inc.), and mouse anti- $\beta$ III tubulin (1:200; Santa Cruz Biotechnology, Inc.) for $2 \mathrm{~h}$ at RT. The cells were washed with PBS three times and incubated with an Alexa Fluor ${ }^{R}$ 488 donkey anti-rabbit IgG $(1: 400)$ or Alexa Fluor ${ }^{\mathbb{R}} 594$ donkey anti-mouse IgG (1:400; Molecular Probes, Eugene, OR, http://www.invitrogen.com) for $40 \mathrm{~min}$ at RT. The cells were also counterstained with 4',6-diamidino-2-phenylindole (DAPI; Vector Laboratories, Burlingame, CA, http://www.vectorlabs.com). Cells were examined using a confocal microscope (Nikon, Yokohama, Japan, http:// www.nikon.com) equipped with a Radiance 2100 MP (Bio-Rad, Hercules, CA, http://www.bio-rad.com) using a Laser Sharp 2000 program (Bio-Rad).

\section{Detection of apoptosis}

For detection of apoptosis, we performed Annexin $\mathrm{V}$ staining by FITC conjugated-Annexin $\mathrm{V}$ following the manufacturer's method (BD Pharmingen, San Diego, CA, http://www.bdbiosciences.com). Briefly, annexin V (FITC-labeled, $5 \mathrm{ml}$ ), propidium iodide $(10 \mathrm{ml})$, and the binding buffer $(400 \mathrm{ml})$ were added to a final volume of 500 $\mathrm{mL}$, and then analyzed by a FACSCalibur flow cytometer (BD Science) within $30 \mathrm{~min}$.

\section{RT-PCR}

Total RNA was extracted using TRIZOL (Invitrogen). Total RNA from each sample was used for first-strand cDNA synthesis by Superscript ${ }^{\mathrm{TM}}$ First Strand Synthesis System for RT-PCR (Invitrogen), according to the manufacturer's instructions. Aliquots of cDNA were used as a template for PCR amplification with specific primer sets. The primer set is listed in Supplemental data Table S1. Ten $\mu$ of PCR products were separated on a $2 \%$ agarose gel and visualized by ethidium bromide staining. The relative expression levels of the detected genes from these cells were estimated visually by comparing relative band intensities with the level of expression of the housekeeping gene, GAPDH.

\section{Western blot analysis}

For Western blot analysis, hESCs were harvested with 2.5 $\mathrm{mg} / \mathrm{ml}$ of collagenase IV (Invitrogen) and washed with PBS. The cells were lysed in ice-cold lysis buffer containing $150 \mathrm{mM}$ Tris- $\mathrm{HCl}(\mathrm{pH} 7.4), 1 \%$ Triton X-100, 25
$\mathrm{mM}$ sodium fluoride (NaF), $80 \mathrm{mM}$ beta-glycerophoshpate, $25 \mathrm{mM} \mathrm{Na} \mathrm{VO}_{4}$, and $1 \%$ phosphatase inhibitor cocktail (Sigma-Aldrich). The cell lysates were incubated on ice for $30 \mathrm{~min}$ and centrifuged at $10,000 \mathrm{~g}$ for $30 \mathrm{~min}$ at $4^{\circ} \mathrm{C}$. After equalizing the protein concentrations, the total lysates were mixed with $\times 5$ sample buffer and boiled for $5 \mathrm{~min}$. All samples were subjected to $9 \%$ or $12 \%$ SDS-PAGE, and electrotransferred onto Hybond $\mathrm{P}$ membranes (Amersham Pharmacia Biotech, Bukinghamshire, UK, http://www. apbiotech.com). The membranes were blocked with $3 \%$ BSA and incubation with primary antibodies were carried out at $4^{\circ} \mathrm{C}$ overnight using rabbit monoclonal antibodies directed against ERK1/2 (1:1000; Santa Cruz Biotechnology, Inc.), anti-JNK (1:1000), anti-p-JNK (1:1000), anti-p38MAPK (1:1000), anti-p-p38MAPK (1:1000), anti-AKT (1:1000), and anti-p-AKT (1:1000), which were all from Cell Signaling Technology (Boston, MA, http://www.cellsignal. com), and mouse monoclonal anti-p-ERK1/2 (1:1000; Santa Cruz Biotechnology, Inc.). After washing, the membranes were incubated with the respective HRP-conjugated secondary antibody for $1 \mathrm{~h}$ at room temperature. Protein expression was detected by enhanced peroxidase detection (EPD; ELPIS BIOTHECH, INC., Taejeon, Korea, http://www.elpis-biotech.co.kr) and protein bands were visualized by film exposure. GAPDH was used as an internal control.

\section{Statistics}

The data are presented as the mean and standard error of the mean. Pairwise testing for the difference between sample means was done with a two-tailed $t$-test. The general linear model procedure was used to analyze more than two independent samples that were further sub-grouped by an additional variable factor. To obtain information regarding the differences among specific group means, Student's $t$-test was used to perform multiple pairwise comparisons. The statistical significance was assigned to the 0.05 level.

\section{Supplemental data}

Supplemental Data include a table and can be found with this article online at http://e-emm.or.kr/article/article_files/SP42-3-03.pdf.

\section{Acknowledgements}

This research was supported by a grant (SC-1150) from the Stem Cell Research Center of the 21st Century Frontier Research Program, funded by the Ministry of Education, Science, and Technology of the Republic of Korea.

\section{References}

Allen RG, Tresini M. Oxidative stress and gene regulation. Free Radic Biol Med 2000;28:463-99

Armstrong L, Hughes O, Yung S, Hyslop L, Stewart R, Wappler I, Peters H, Walter T, Stojkovic P, Evans J, Stojkovic 
$\mathrm{M}$ and Lako $\mathrm{M}$. The role of PI3K/AKT, MAPK/ERK and NFkappabeta signalling in the maintenance of human embryonic stem cell pluripotency and viability highlighted by transcriptional profiling and functional analysis. Hum Mol Genet 2006;15:1894-913

Ateghang B, Wartenberg M, Gassmann M, Sauer H. Regulation of cardiotrophin-1 expression in mouse embryonic stem cells by HIF-1alpha and intracellular reactive oxygen species. J Cell Sci 2006;119:1043-52

Buggisch M, Ateghang B, Ruhe C, Strobel C, Lange S, Wartenberg M, Sauer H. Stimulation of ES-cell-derived cardiomyogenesis and neonatal cardiac cell proliferation by reactive oxygen species and NADPH oxidase. J Cell Sci 2007;120:885-94

Bunting KD. ABC transporters as phenotypic markers and functional regulators of stem cells. Stem Cells 2002;20:11-20

Burdon T, Smith A, Savatier P. Signalling, cell cycle and pluripotency in embryonic stem cells. Trends Cell Biol 2002;12:432-8

Carpenter MK, Rosler ES, Fisk GJ, Brandenberger R, Ares $X$, Miura T, Lucero M and Rao MS. Properties of four human embryonic stem cell lines maintained in a feeder-free culture system. Dev Dyn 2004;229:243-58

Ding L, Liang XG, Hu Y, Zhu DY and Lou YJ. Involvement of p38MAPK, reactive oxygen species in icariin-induced cardiomyocyte differentiation of murine embryonic stem cells in vitro. Stem Cells Dev 2008;17:751-60

Dvorak P, Dvorakova D, Koskova S, Vodinska M, Najvirtova M, Krekac D, Hampl A. Expression and potential role of fibroblast growth factor 2 and its receptors in human embryonic stem cells. Stem Cells 2005;23:1200-11

Ito K, Hirao A, Arai F, Takubo K, Matsuoka S, Miyamoto K, Ohmura M, Naka K, Hosokawa K, Ikeda Y, Suda T. Reactive oxygen species act through p38 MAPK to limit the lifespan of hematopoietic stem cells. Nat Med 2006;12: 446-51

Lange S, Heger J, Euler G, Wartenberg M, Piper HM, Sauer $\mathrm{H}$. Platelet-derived growth factor BB stimulates vasculogenesis of embryonic stem cell-derived endothelial cells by calcium-mediated generation of reactive oxygen species. Cardiovasc Res 2009;81:159-68

Oh SK, Kim HS, Ahn HJ, Seol HW, Kim YY, Park YB, Yoon CJ, Kim DW, Kim SH, Moon SY. Derivation and characterization of new human embryonic stem cell lines:
SNUhES1, SNUhES2, and SNUhES3. Stem Cells 2005; 23:211-9

Park YB, Kim YY, Oh SK, Chung SG, Ku SY, Kim SH, Choi YM, Moon SY. Alterations of proliferative and differentiation potentials of human embryonic stem cells during long-term culture. Exp Mol Med 2008;40:98-108

Rhee SG. Redox signaling: hydrogen peroxide as intracellular messenger. Exp Mol Med 1999;31:53-9

Sauer H, Rahimi G, Hescheler J, Wartenberg M. Effects of electrical fields on cardiomyocyte differentiation of embryonic stem cells. J Cell Biochem 1999;75:710-23

Sauer H, Rahimi G, Hescheler J, Wartenberg M. Role of reactive oxygen species and phosphatidylinositol 3-kinase in cardiomyocyte differentiation of embryonic stem cells. FEBS Lett 2000;476:218-23

Sauer $\mathrm{H}$, Wartenberg $M$. Reactive oxygen species as signaling molecules in cardiovascular differentiation of embryonic stem cells and tumor-induced angiogenesis. Antioxid Redox Signal 2005;7:1423-34

Shibanuma M, Kuroki T and Nose K. Stimulation by hydrogen peroxide of DNA synthesis, competence family gene expression and phosphorylation of a specific protein in quiescent Balb/3T3 cells. Oncogene 1990;5:1025-32

Simon AR, Rai U, Fanburg BL, Cochran BH. Activation of the JAK-STAT pathway by reactive oxygen species. Am J Physiol 1998;275:C1640-52

Sohal RS, Allen RG, Nations C. Oxygen free radicals play a role in cellular differentiation: an hypothesis. J Free Radic Biol Med 1986;2:175-81

Tada S, Era T, Furusawa C, Sakurai H, Nishikawa S, Kinoshita M, Nakao K, Chiba T. Characterization of mesendoderm: a diverging point of the definitive endoderm and mesoderm in embryonic stem cell differentiation culture. Development 2005;132:4363-74

Thannickal VJ, Fanburg BL. Reactive oxygen species in cell signaling. Am J Physiol Lung Cell Mol Physiol 2000; 279:L1005-28

Valko M, Leibfritz D, Moncol J, Cronin MT, Mazur M, Telser $\mathrm{J}$. Free radicals and antioxidants in normal physiological functions and human disease. Int $\mathrm{J}$ Biochem Cell Biol 2007;39:44-84 